\title{
Bifunctional Tripeptide Including a Phosphonic Acid as Brønsted Acid for Michael Addition: Mechanistic Insights
}

\author{
Margery Cortes-Clerget,${ }^{[a]}$ Jesús Jover, ${ }^{[b][c]}$ Jade Dussart, ${ }^{[a]}$ Emilie Kolodziej, ${ }^{[\mathrm{d}]}$ Maelle Monteil, ${ }^{[\mathrm{a}]}$ \\ Evelyne Migianu-Griffoni, ${ }^{[a]}$ Olivier Gager,${ }^{[\mathrm{a}]}$ Julia Deschamp, ${ }^{\left[{ }^{[a]}\right.}$ Marc Lecouvey ${ }^{*[a]}$
}

\begin{abstract}
Enamine catalysis is a widespread activation mode in the field of organocatalysis and is often encountered in bifunctional organocatalysts. We previously described H-Pro-Pro-pAla-OMe as a bifunctional catalyst for Michael addition between aldehydes and aromatic nitroalkenes. As opposite selectivities were observed compared to its analogue $\mathrm{H}-$ Pro-Pro-Glu- $\mathrm{NH}_{2}$ described by Wennemers, its activation mode was investigated through kinetic, linear effect studies, NMR analyses and structural modifications. It appeared that only one bifunctional catalyst was involved in the catalytic cycle, by activating aldehyde through an $(E)$-enamine and nitroalkene via an acidic interaction. A restrained tripeptide structure was optimal in terms of distance and rigidity for better selectivities and fast reaction rates. Transition state modeling unveiled the particular selectivity of this phosphonopeptide.
\end{abstract}

Combining either amino-, H-bonding or Lewis base catalysis, hydrophobic effect and in-solvent organization, they were early introduced to mimic enzyme behavior and selectively interact with substrates. ${ }^{[7]}$ Beyond the structural peptide complexity, Miller has recently proposed combinatorial screenings to easily identify novel peptide-based catalysts to promote enantioselective processes. $^{[8]}$ Afterwards Wennemers has highlighted the tripeptides $\mathrm{H}$-Pro-Pro-Asp- $\mathrm{NH}_{2}$ and H-Pro-ProGlu- $\mathrm{NH}_{2}$ as efficient catalysts for enantioselective aldol reaction and Michael addition, respectively (Scheme 1$).{ }^{[5 d, 9]}$
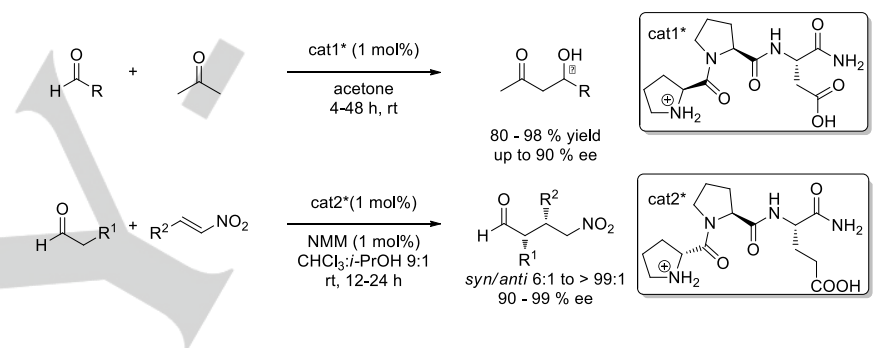

Scheme 1. Wennemers' catalysts effective on aldolisation and 1,4-addition reactions a subject of interest especially in terms of stereospecificity and stereoselectivity. Inspiring by enzyme structures, organic chemists aim at developing more efficient, selective and sustainable catalysts. Despite the use of proline as catalyst has been known since the 70 s, organocatalysis ${ }^{[1]}$ has trully emerged as a powerful tool for asymmetric transformation since the independent works of Barbas, Lerner, List and MacMillan in $2000 .^{[2]}$ Nowadays, organocatalysis via covalent ${ }^{[3]}$ or noncovalent $^{[4]}$ activations enables the selective formation of one or several stereogenic centers ${ }^{[5]}$ at once. Moreover these methods have been successfully employed for the synthesis of biologically active compounds. ${ }^{[6]}$ Thanks to their multifunctional structure, amino acids and peptides are especially efficient at multiactivating easily accessible or natural molecules.

[a] Dr. M. Cortes-Clerget, Dr. O. Gager, Dr. M. Monteil, Dr. E. MigianuGriffoni, J. Dussart, Dr. J. Deschamp and Prof. M. Lecouvey Université Paris 13, Sorbonne Paris Cité - Laboratoire CSPBAT CNRS UMR 7244, 1 Rue de Chablis, 93000 Bobigny - France E-mail: marc.lecouvey@univ-paris13.fr

E-mail: julia.deschamp@univ-paris13.fr

[b] Dr. J. Jover, Institute of Chemical Research of Catalonia (ICIQ), The Barcelona Institute of Science and Technology, Avgda. Països Catalans, 16, 43007 Tarragona, Spain

[c] Departament de Química Inorgànica i Orgànica, Secció de Química Inorgànica, Universitat de Barcelona, Martí i Franquès 1-11, 08028 Barcelona, Spain

[d] E. Kolodziej, Université Paris Sud, ICMMO, UMR 8182, 15 Rue Georges Clemenceau 91405 Orsay Cedex

Supporting information for this article is given via a link at the end of the document.
Additionally, the same team showed that multiple modes of catalyst activation are required, where the absence of either the $\mathrm{N}$-terminal secondary amine, or the carboxylic acid inhibited the reaction. They also provided evidence for an enamine rather than an enol mechanism by ESI-MS investigations. ${ }^{[10]}$

Our group have recently reported the first example of H-Pro-PropAla-OMe ${ }^{[11]}$ combining both aminocatalysis and phosphonic acid-activation for the diastereo- and enantioselective conjugate addition of aldehydes onto nitroalkenes. ${ }^{[12]}$ We developed a straightforward synthesis of the modified amino-acids and their of the three stereogenic centers and the lateral chain length of the amino-acid bearing the phosphonic acid moiety (Figure 1).

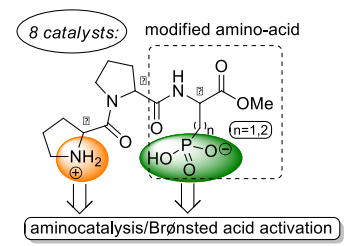

Figure 1. Novel bifunctional catalysts including a phosphonic acid moiety

Our preliminary studies focused on a Michael addition as a model reaction between aldehydes and nitroalkenes. Among our catalyst library, the tripeptide $\mathbf{l a}_{11.5^{[13]}}$ enabled us to obtain the corresponding tripeptides by varying the absolute configuration 
corresponding $\gamma$-nitroaldehydes in excellent yields and with good to excellent stereoselectivities (up to 95:5 d.r.; 93:7 e.r.) without any external base or side product (Scheme 2 ).

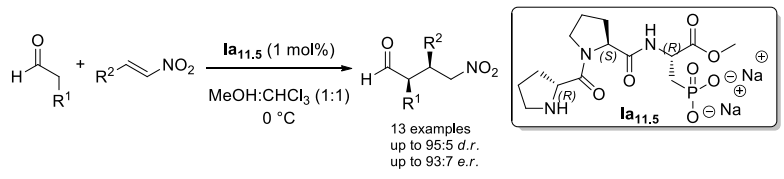

Scheme 2. Michael addition between aldehydes and aromatic nitroalkenes catalyzed by $\mathbf{l a}_{11.5}$

In order to propose a sustainable process, the catalyst recycling was performed. Thanks to their high water solubility, these catalysts were easily recovered by simple extractions and lyophilization. Several recycling tests were achieved without any significant loss of selectivities (10 times). Owing to the phosphonic acid group, our tripeptides have the advantage to be directly recyclable and thus, they do not require to be immobilized on a solid support.

In our former work, the dependence of various parameters such as catalyst loading and protonated-state catalyst were thoroughly explored. Surprisingly, for a given spatial configuration catalyst, we obtained the opposite products when compared to Wennemers' catalyst (Scheme 3).

$$
\text { (S) catalysts }
$$

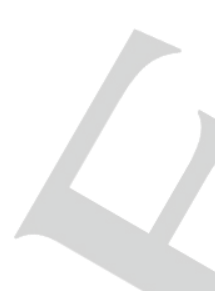

Scheme 3. Wennemers' catalyst vs. la I1.5: $_{1.5}$ opposite selectivities

As our research focused on the impact of a phosphonic acid compared to a carboxylic acid on both reactivity and selectivities, we have started some mechanistic investigations to understand our previous observations. Herein, we disclose our results in order to highlight the potential of our novel tripeptide-catalysts.

\section{Results \& Discussion}

The first experiment was intended to elucidate which functional groups were required for asymmetric catalysis (Scheme 4). The reaction was carried out between propanal and trans- $\beta$ nitrostyrene in presence of the $\mathrm{N}$-Boc-protected tripeptide Bocla in the optimized conditions that we previously reported. ${ }^{[12]}$ The ionization state of the phosphonic salt was pre-adjusted to $\mathrm{pH}=$ 7.5 with a sodium hydroxide solution during the catalyst preparation. As expected, no reaction occurred, demonstrating that the amine group is essential for activation. In the same manner, the phosphonic acid was replaced by a phosphonate. Even with the terminal secondary amine-free proline, catalyst la$(\mathrm{OMe})_{2}$ was significantly poorer than $\mathrm{la}_{11.5}$. The reaction rate was very slow (conversion $<10 \%$ after $48 \mathrm{~h}$ ) and the selectivities dropped dramatically (syn:anti 66:34 and $32 \%$ e.r.).

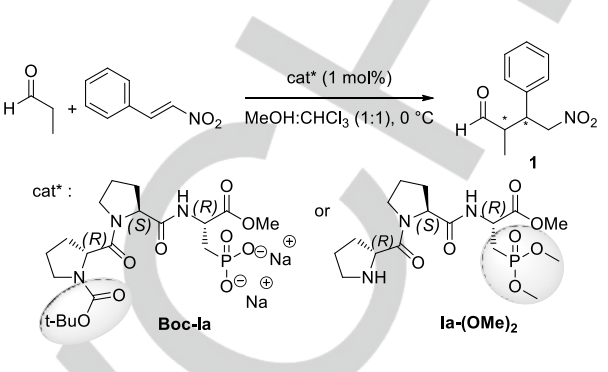

Scheme 4. Michael addition between propanal and trans- $\beta$-nitrostyrene catalyzed by tripeptides Boc-la or la-(OMe $)_{2}$

In view of these results, we conclude that both the secondary amine and the phosphonic acid groups are essential to keep good reactivity, diastereo- and enantioselectivities.

After that, 15 diastereoisomers of la were prepared to investigate the variations on both catalyst configurations and side chain length holding the phosphonic acid group. ${ }^{[14]}$ The catalysts were screened in the presence of propanal and trans$\beta$-nitrostyrene under the optimized conditions (Figure 2). The reactivity of the catalysts was excellent as total conversion was achieved within less than 6 hours for all the evaluated diastereoisomers. The selectivities varied accordingly to the catalytic pocket geometry. Changing the terminal proline absolute configuration can significantly reduce the enantiomeric (e.g. Ild $\rightarrow \mathrm{Ila}$ - row 2) and the diastereoisomeric ratios (e.g. Id $\rightarrow$ Ic - row 1). Moreover, opposite absolute configurations were obtained for product 1 (Figure 2 - red and green).

Increasing the side chain length (Figure 2 - columns) extended the time to completion and reduced the diastereoisomeric ratios indicating that higher flexibility implies lower selectivities. All these results showed the specific $\mathrm{H}-R$-Pro-S-Pro- $R$-pAla-OMe structure was crucial for a good selectivity but also for a fast reaction in an intramolecular way. In our former works, we also noted the importance of the protonated-state of the catalyst as better selectivities were observed when the tripeptide catalyst was pre-adjusted at $\mathrm{pH}=11.5$.

Since the geometry of the catalytic pocket is crucial for a good stereoselectivity, we focused our interest on studying the importance of the central proline for selectivities (Table 1).

We started replacing the central proline by a less bulky and more flexible amino acid like glycine, obtaining catalyst le that was then evaluated in standard conditions (Table 1 - entry 2 ). The diastereoselectivity dropped ((65:35 vs. $91: 9$ d.r.) and a lower enantioselectivity was observed (23:77 vs. 93:7 e.r.) although the reaction time was extended ( $35 \mathrm{~h}$ vs. $20 \mathrm{~h}$ ). The rigidity imposed by the proline residue seems to be crucial for an efficient diastereo-discrimination. As expected, the opposite enantiomer product $1(2 S, 3 R)$ was obtained as the enantiomer catalyst le was used (Table 1 - entries 2 vs. 1 ). 


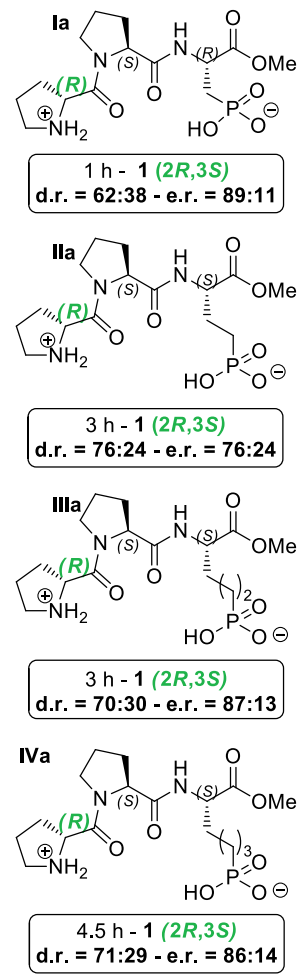

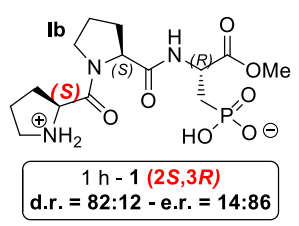
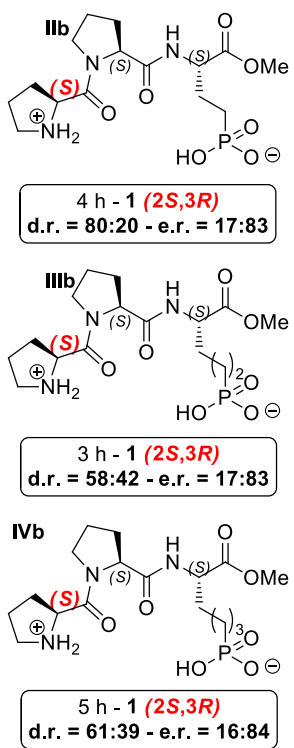
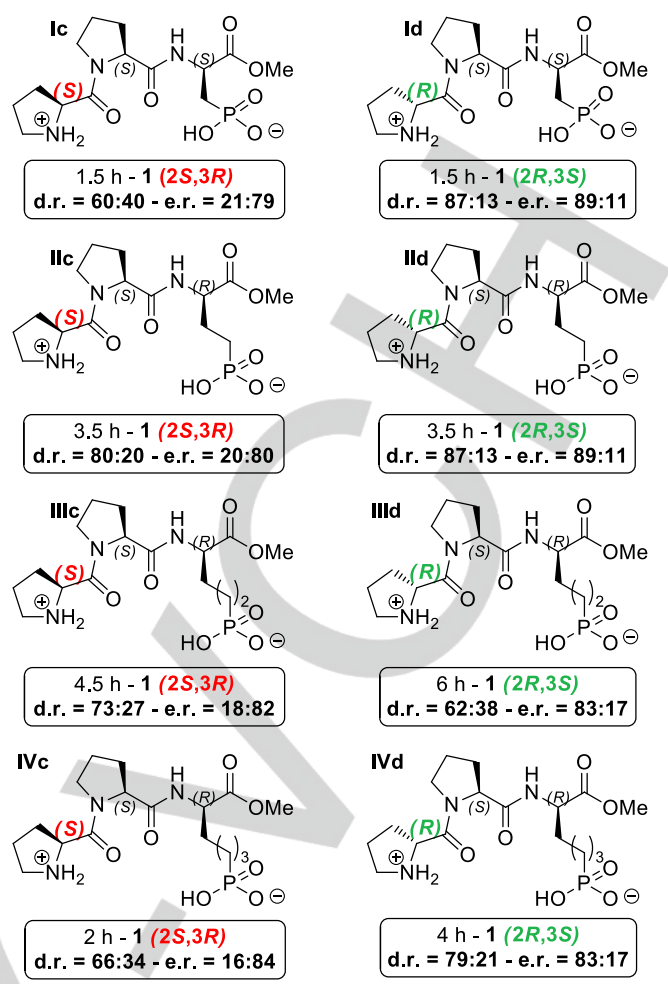

$3.5 \mathrm{~h}-1(2 R, 3 S)$
d.r. $=87: 13-$ e.r. $=89: 11$
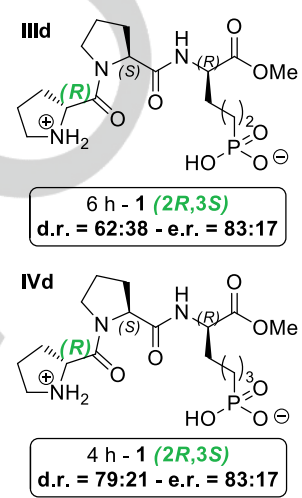

Figure 2. Influence of catalyst I-IV absolute configurations and lateral chain lengths on 1,4-conjugated addition of propanal onto trans- $\beta$-nitrostyrene

After that, the central proline was suppressed and dipeptides If and Ig were tested but, no total conversion was reached after a week (Table 1 - entries 3 and 4). As predicted by Wennemers, ${ }^{\text {[a] }}$ the catalytic pocket of these compound might be too small for a $\beta$-position attack and consequently an intermolecular reaction probably occurred with a drop of selectivities. However, it was noted that better selectivities were obtained in presence of Ig rather than If. The link between the two activating functions was then suppressed in order to investigate the synergic catalysis; it was done by replacing the tripeptide by amino-acid "cocktails". It was firstly noted that modified amino acid $\mathbf{A A}_{1}$ alone gave less than $1 \%$ conversion after 5 days (Table 1 - entry 5 ). The primary amine appears not to be nucleophilic enough to form an enamine with propanal. Proline alone allowed the formation of the $\gamma$-nitroaldehyde $\mathbf{1}$ after a long reaction time with a low enantioselectivity (Table 2 - entry 6 ). A mixture of proline and amino acid $\mathbf{A A}_{\mathbf{1}}$, named cocktail $\mathbf{A}(1: 1)$ and a mixture of H-ProOMe and $N$-Boc-protected $\mathbf{A A}_{1}$, called cocktail $\mathbf{B}$ (1:1) were evaluated under the optimized conditions (Table 1 - entries 7 and 8 ). We observed that the reaction rates were 6 times slower for both mixtures. The diastereoisomeric ratio slightly decreased for cocktail A while remained the same for cocktail B. In both cases, the enantiomeric ratios were lower compared to those obtained with la $\mathbf{a}_{11.5}$. Although these two cocktails have the same absolute configurations, interestingly, the opposite products were obtained. We suppose that this discrepancy arises from the difference between the transition states leading to the formation of the corresponding products (Figure 3).
Table 1. Influence of the catalyst structure.

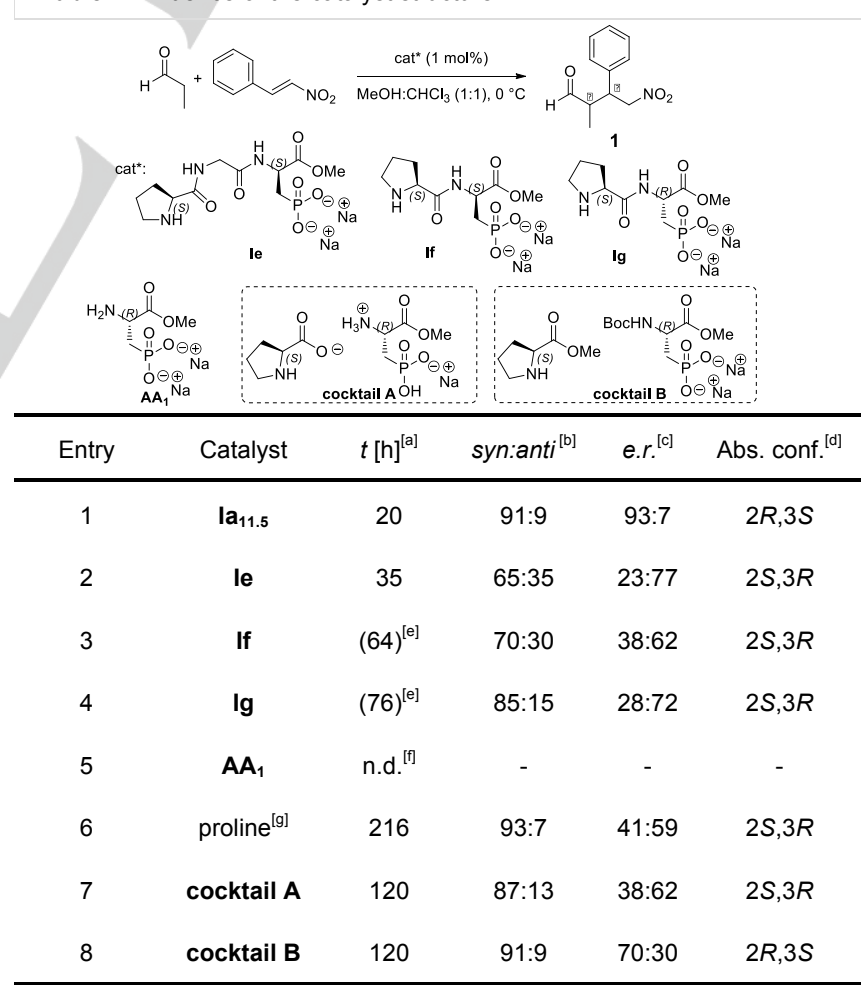

[a] Time to completion, estimated by GC analysis. [b] Determined by ${ }^{1} \mathrm{H}$ NMR spectroscopy on the crude material. [c] e.r. of the syn-adduct were determined by chiral-HPLC analysis. [d] Compared to optical rotation given in literature. [e] Expressed in conversion rate after a week. [f] Less than $1 \%$ of conversion after a week. [g] The reaction was conducted at $\mathrm{pH}=11.5$ 
In the case of cocktail A, an electrostatic interaction allows the self-assembly of the amino-acids while for cocktail $\mathbf{B}$ the steric bulkiness of the dangling tert-butyl group pulls away the two partners. The same enantioselectivity was obtained with cocktail $\mathbf{A}$ and catalyst If but the diastereoisomeric ratio is higher for the former (Table 1 - entries 3 vs 7). Cocktail B and catalyst $\mathbf{l a}_{11.5}$ gave the same diastereoselectivity albeit a lower enantiomeric ratio was obtained with cocktail B (Table 1 entries 1 vs 8 ).

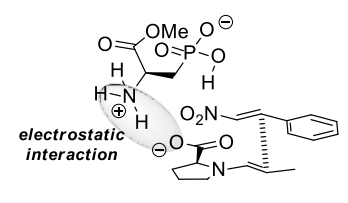

cocktail A

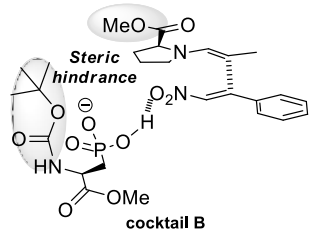

Figure 3. Postulated transition states involving cocktail A and cocktail B

After studying the importance of the catalyst structure, we decided to explore the catalyst activation mode. According to the literature, ${ }^{[15]}$ the postulated mechanism should involve the formation of an $E$-enamine between the secondary amine group and the aldehyde (Scheme 5). Then, the nitroalkene activation through an acidic interaction should increase the electrophilicity of the $\beta$-carbon and allow the enamine attack. Thanks to the geometry of the catalytic pocket, the $\mathrm{C}-\mathrm{C}$ bond could be created in a stereoselective way. The final hydrolysis should concomitantly permit the liberation of the expected $\gamma$-nitroaldehyde and regenerate the active catalyst.

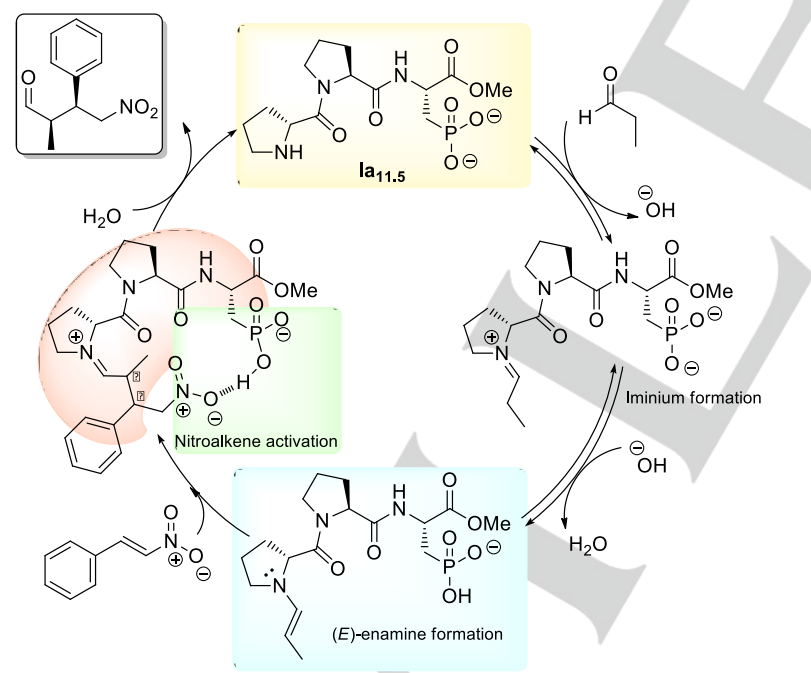

Scheme 5. Postulated catalytic cycle for the la $\mathbf{a}_{11.5}$-catalyzed 1,4-addition reaction

In order to validate the involvement of only one molecule of catalyst, kinetic studies and non-linear effect experiments were conducted in presence of catalyst la. The 1,4-addition between propanal and trans- $\beta$-nitrostyrene was catalyzed at $1,2.5,5,7.5$ and $10 \mathrm{~mol} \%$ of la at $\mathrm{pH}=6.6$ or 11.5 . The partial order with regard to the catalyst was respectively 0.8 and 0.9 at $\mathrm{pH}=6.6$ and $11.5 .{ }^{[16]}$ Both these values are close to 1 , indicating that the rate of the reaction increases linearly with the catalyst loading. Although Kagan and Agami had observed a slight non-linear effect on the proline-catalyzed Hajos-Parrish reaction. ${ }^{[17]}$ List $^{[18]}$ contradicted these results by providing evidences for the involvement of only one pyrrolidine in the transition state of intraand intermolecular aldol reaction. ${ }^{[19]}$ To verify the linear relationship between the enantiomeric excess of the catalyst and the product, the reaction above was catalyzed by a mixture of la and its enantiomer in several ratios at 0 or $25{ }^{\circ} \mathrm{C}$, with catalyst loadings of 1 or $10 \mathrm{~mol} \%$ and pre-adjusted $\mathrm{pH}$ values of 6.6 or 11.5. A linear effect was observed regardless of the experimental conditions. ${ }^{[20]}$ Both kinetic studies and non-linear effect experiments provide proofs that only one molecule of catalyst was involved in the catalytic process.

NMR experiments were performed to possibly identify some iminium/enamine intermediates. The catalyst la was mixed with an excess of propanal in a mixture of chloroform-d: methanol- $\mathrm{d}_{4}$ (1:1). The signal of propanal $(9.44 \mathrm{ppm})$ decreased and two new signals were detected at $6.26 \mathrm{ppm}$ and $9.04 \mathrm{ppm}$ which could correspond to enamine and iminium intermediates (Figure 4). ${ }^{[21]}$
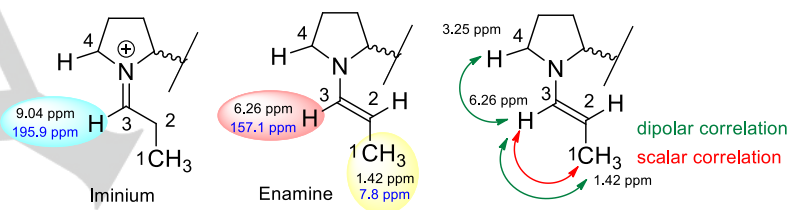

Figure 4. Identification of iminium and $(E)$-enamine by ${ }^{1} \mathrm{H}$ (in black) and ${ }^{13} \mathrm{C}$ NMR (in blue)

2D NMR studies were conducted to confirm the nature of the new products. COSY experiment highlighted the scalar correlation between the enamine proton (3) $(6.26 \mathrm{ppm})$ and the $\mathrm{CH}_{3}$ group (1) (1.42 ppm).

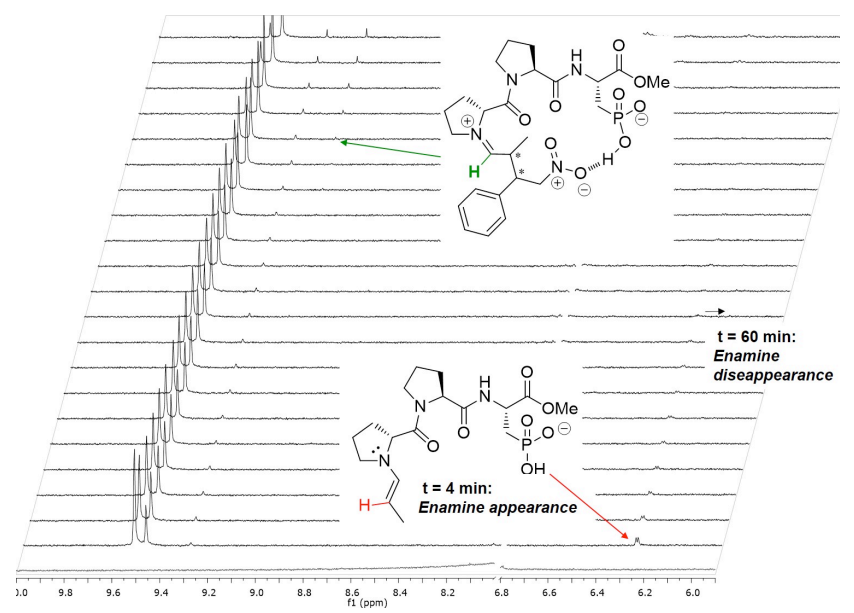

Figure 5. Enamine/iminium formation followed by ${ }^{1} \mathrm{H}$ NMR 
Table 2. Influence of the $\mathrm{pH}$ and counter-cation nature in catalysis

(1)

[a] Time to completion, estimated by GC analysis. [b] Determined by $1 \mathrm{H}$ NMR spectroscopy of the crude material. [c] The e.r. of the syn-adduct were determined by chiral-phase HPLC analysis. [d] The reaction was carried out in presence of 2 equiv. of trimethylamine.

Moreover, NOESY showed a dipolar correlation with the $\alpha$ proton (4) of the pyrrolidine (3.25 ppm) (Figure 4). These observations confirm the formation of an enamine with an $E$ configuration in agreement with the results reported by Blackmond, who observed an enamine proton at $6.1 \mathrm{ppm} \cdot{ }^{[22]}$ The addition of pre-activated molecular sieves in the NMR tube induced an increasing $6.26 \mathrm{ppm}$ enamine signal at $9.44 \mathrm{ppm}$ propanal signal expense. This observation seems to confirm the existing equilibrium at this step.

The reaction between propanal and trans- $\beta$-nitrostyrene was then carried out in presence of la and monitored by ${ }^{1} \mathrm{H}$ NMR (Figure 5). Enamine intermediate appeared at the first scan at $6.26 \mathrm{ppm}$. This signal rapidly decreased in favor of the formation of a novel signal which could correspond to a second iminium compound (9.2 ppm)

Thereafter, kinetic studies were performed to determine the reaction partial orders with regard to the aldehyde and the nitroalkene in presence of la, initially pre-adjusted at $\mathrm{pH}=6.6$ and $11.5 .^{[13]}$ The partial order of propanal is 0.4 in presence of catalyst $\mathbf{l a}_{6.6}$ and drops to 0.1 for catalyst $\mathbf{l a}_{11.5}$. Concerning trans- $\beta$-nitrostyrene, the partial order is 0.3 in presence of catalyst $\mathbf{l} \mathbf{a}_{6.6}$ and 0.2 for catalyst $1 \mathbf{a}_{11.5}$.
The protonated-state of the catalyst is strongly correlated with the partial orders of the aldehyde while its effect is limited on the nitroalkene partial order. Regardless of the $\mathrm{pH}$ pre-adjusted catalysts, enamine formation seems not to be the rate-limiting step. These kinetic studies corroborate our initial experimental observations about the postulated enamine formation.

Nevertheless, several questions remain concerning the influence of the peptide protonated-state on the catalytic process. We supposed that nitroalkene is activated by the phosphonic acid through an acidic interaction.

However, Ishihara showed that the calcium salt of the BINOLphosphoric acid was as effective as the acidic form itself. ${ }^{[23]}$ In order to identify the real activation mode of our tripeptide la, we studied the influence of its protonated-state (Table 2). The reaction still occurred with the all-deprotonated catalyst $\mathbf{l a}_{11.5}$, while no reaction was observed in presence of $\mathbf{l a}_{2.3}$ (Table 2 entries 2 and 3 ). Moreover, $\mathbf{l a}_{11.5}$ was surprisingly a more reactive and selective catalyst than $\mathbf{l a}_{6.6}$ and the reaction was completed after only $20 \mathrm{~h}$. Nevertheless, when the reaction was conducted between propanal and trans- $\beta$-nitrostyrene in presence of $\mathbf{l a}_{11.5}$, the $\mathrm{pH}$ was monitored during the reaction. A drop of $\mathrm{pH}$ from 11.5 to 6.6 was early observed, which seems to confirm the presence of the acidic specie. We tested the same reaction in presence of $\mathbf{l a}_{11.5}$ and 2 equivalents of triethylamine in order to keep the $\mathrm{pH}$ value around 8-9 (Table 2 - entry 4). Here, the reaction rate dramatically dropped and slightly lower selectivities were obtained. This observation provides the evidence that an acidic hydrogen on the phosphonic acid should be responsible for the nitroalkene activation, through a hydrogen bonding or ion-pairing.

To confirm the previous observations, the nature of the countercation was investigated by replacing the sodium salt by its lithium or calcium analogues (Table 2). The lithium salt la' ${ }_{11.5}$ gave the same results whereas the calcium salt la" 11.5 showed poor reactivity ( $20 \mathrm{~h}$ vs. $192 \mathrm{~h}$ ). It is not surprising that lithium which is quite similar to sodium, does not modify the catalytic pocket. On the other hand, calcium is bigger and divalent and probably disrupts the peptide conformation.

Based on our experiments, we propose the revised catalytic cycles in order to include the $\mathrm{pH}$ effect (Scheme 6). By comparing the initial rate for each protonated-state, it appears the reaction starts faster in the presence of $\mathbf{l} \mathbf{a}_{11.5}$ rather than $\mathbf{l} \mathbf{a}_{6.6}$ (Figure 6).

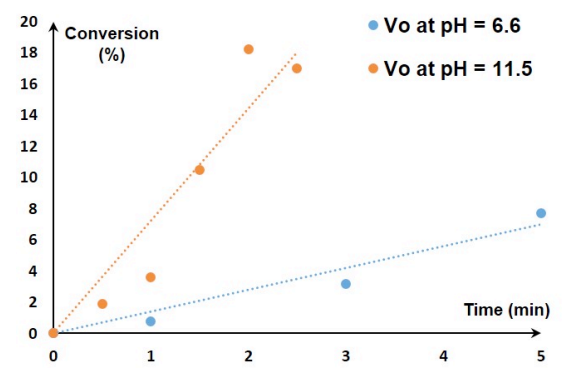

Figure 6. Comparison of initial rates depending on the catalyst protonatedstate 


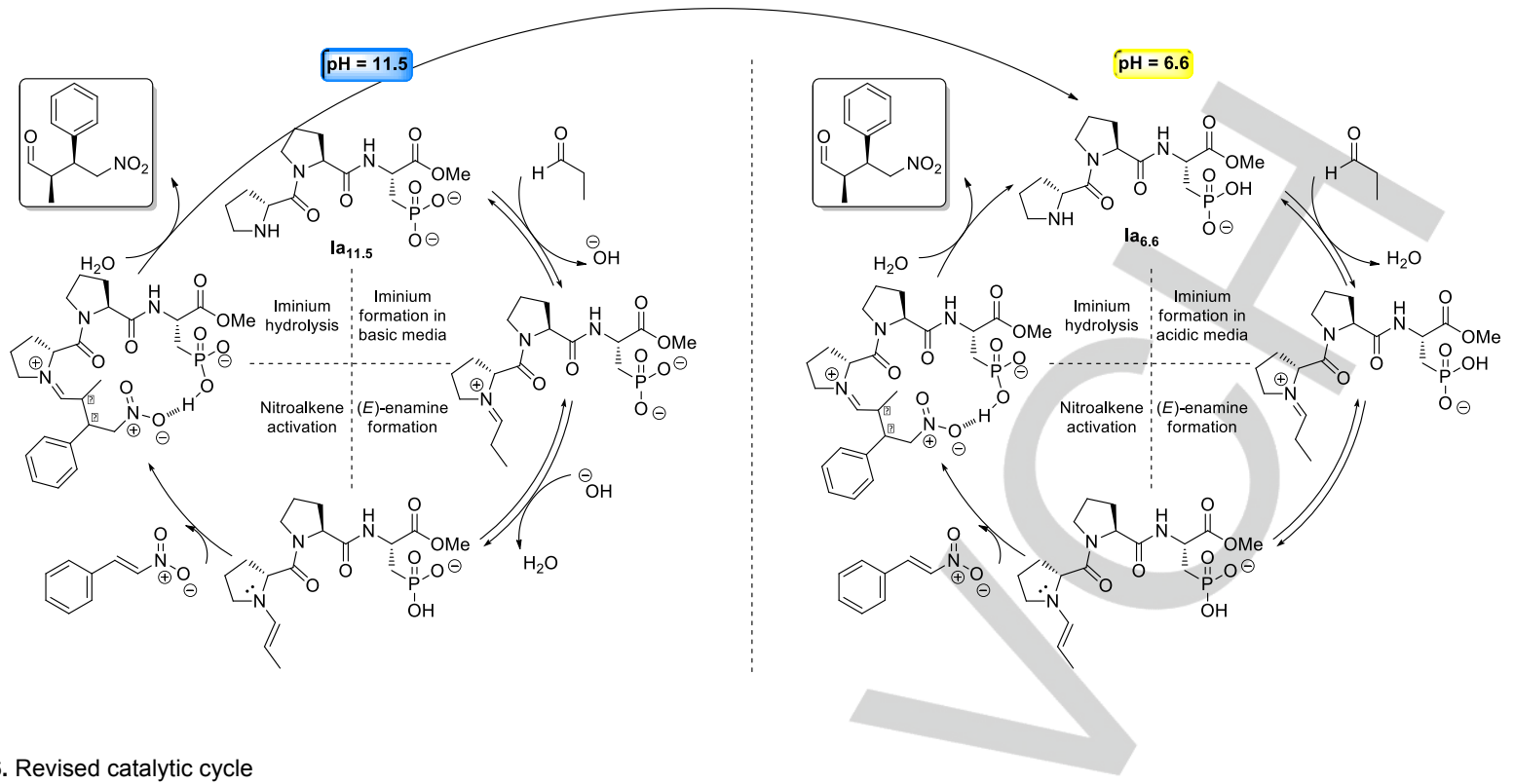

Scheme 6. Revised catalytic cycle

The catalyst $\mathbf{l}_{\mathbf{1 1 . 5}}$ already has the reactive amine on the terminal proline that could readily enable the formation of the iminium and then, enamine intermediates with propanal.

At this stage, the catalyst could be protonated allowing the nitroalkene $\mathrm{H}$-bonding activation. The second iminium formation in presence of water could concomitantly release the expected $\gamma$-nitroaldehyde and regenerate the catalyst $\mathbf{l a}_{6.6}$ that permits the catalytic process to proceed.

Thereafter, the reaction was performed in presence of activated molecular sieves. The achieved conversion was never higher than $10 \%$. Besides, the 1,4-addition was carried out between propanal and trans- $\beta$-nitrostyrene in presence of $\mathbf{l a}_{11.5}$ and water, and no conversion was obtained. These results confirmed the harmful effect of water excess in the catalytic cycle, notably for the enamine formation. However the released water during the enamine formation is essential because it allows the second iminium hydrolysis. These observations support the formation of all the iminium/enamine intermediates.

Density functional theory (DFT) theoretical studies have been carried out in order to explain the observed enantioselectivity with peptide la. ${ }^{[20]}$ The four transition states determining the final stereochemistry of the product i.e. syn $(2 R, 3 S)$, anti $(2 R, 3 R)$, syn $(2 S, 3 R)$ and anti $(2 S, 3 S)$, in the reaction between propanal and trans- $\beta$-nitrostyrene with peptide $\mathbf{l a}_{6.6}$ were computed. Their relative free energy barriers for the $(E)$-enamine attack onto trans- $\beta$-nitrostyrene are $4.5,4.9,9.4$ and $10.8 \mathrm{kcal} / \mathrm{mol}$ for the above products, respectively (Scheme 7 ).

These results clearly indicate that product syn $(2 R, 3 S)$ is the most favored reaction outcome, in a perfect agreement with our NMR experiments. The free energy difference between the syn $(2 R, 3 S)$ and anti $(2 R, 3 R)$ diastereoisomers also agrees with the experimentally observed proportions, a difference of 0.4 $\mathrm{kcal} / \mathrm{mol}$ at room temperature should roughly produce a selectivity ratio of 70:30, not very far of that observed experimentally. Figure 7 shows the transition states leading to these products and includes some selected bond distances.

The $\mathrm{C}-\mathrm{C}$ bond distances in these transition states are quite long: 2.41 and $2.24 \AA$ for the syn- and anti-species respectively, indicating the early character of these structures. As may be observed, the presence of the sodium cation actively participates in the reaction by binding the nitro group of the substrate, the $\mathrm{Na}$-Onitro distances are always shorter than $2.5 \AA$. As predicted in our experiments, the binding between trans- $\beta$-nitrostyrene and the peptide is reinforced by the hydrogen bond interaction established between the nitro and phosphoric groups, which show very short distances in both cases (ca. $1.85 \AA$ ).

The alternative syn $(2 S, 3 R)$ and anti $(2 S, 3 S)$ products are obtained in a much lower extent because they require the formation of a starting ( $Z$ )-enamine between la and propanal. This process is highly unlikely since this latter species is almost $10 \mathrm{kcal} / \mathrm{mol}$ higher than its $(E)$-counterpart.
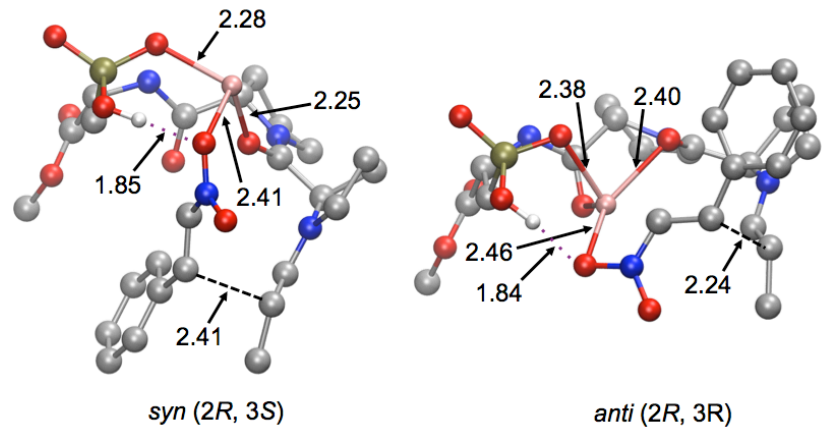

Figure 7. Transition states leading to the major products (distances in $\AA$, color code: $\mathrm{C}=$ gray, $\mathrm{N}=$ blue, $\mathrm{O}=$ red, $\mathrm{P}=\tan , \mathrm{Na}=$ pink, $\mathrm{H}=$ white; for clarity most $\mathrm{H}$-atoms have been omitted) 

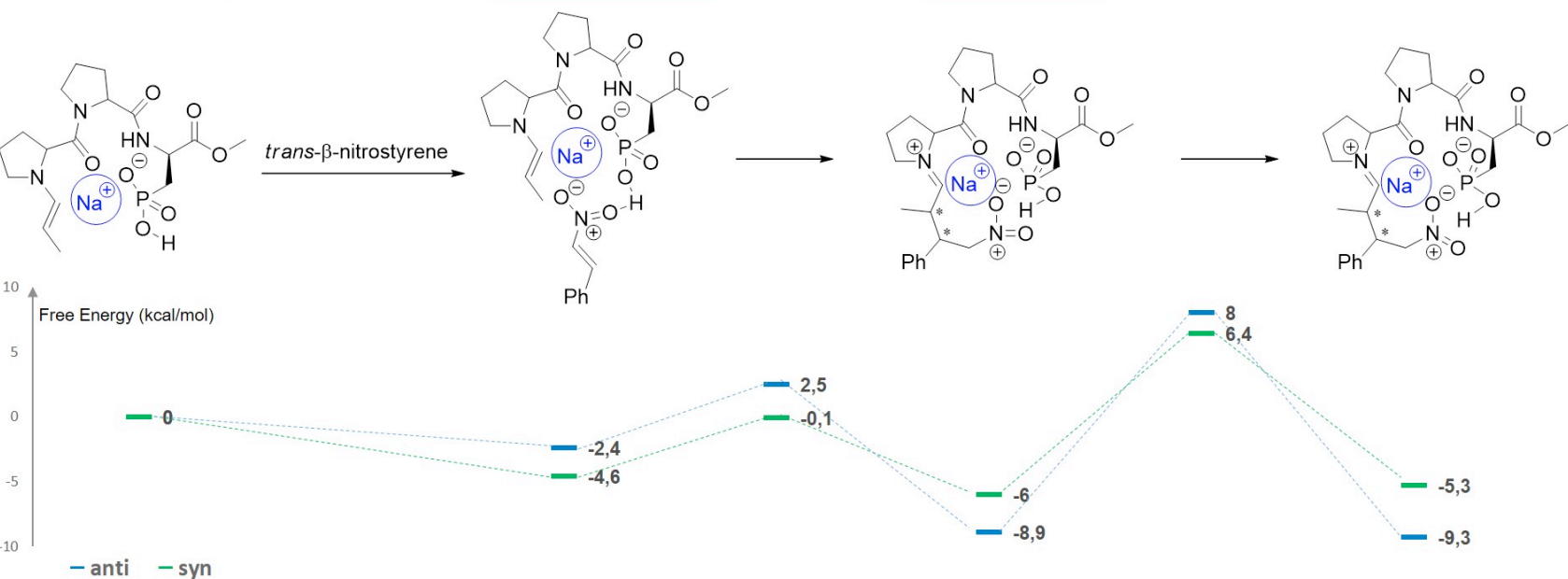

Scheme 7. Computed free energy profiles $(\mathrm{kcal} / \mathrm{mol})$ for the reaction of trans- $\beta$-nitrostyrene with the propanal $(E)$-enamine of peptide la 6.6 leading to the syn $(2 R$, $3 S)$ and anti $(2 R, 3 R)$ products

The free energy profiles describing the reaction of trans- $\beta$ nitrostyrene with the propanal $(E)$-enamine of peptide $\mathbf{l a}_{6.6}$ are shown in Scheme 7. As expected, the barrier for the formation of the syn $(2 R, 3 S)$ product remains lower than that for the obtention of the anti $(2 \mathrm{R}, 3 \mathrm{R})$ analogue: 12.4 and $16.9 \mathrm{kcal} / \mathrm{mol}$, respectively. These calculations show also that the highest free energy barriers of the reaction are not those determining the final product configuration but the ones corresponding to the protonation after the new $\mathrm{C}-\mathrm{C}$ bond formation.

The computed barriers are, however, too low to account for the observed reaction rate. This is probably due to a different reaction stage e.g. the $(E)$-enamine formation or the final product release, not directly related to the enantioselection process, has a higher free energy barrier in the overall reaction pathway.

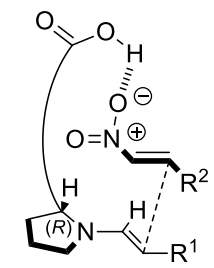

Wennemers' catalyst

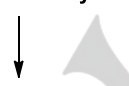<smiles>[R]C(C=O)C([R])C[N+](=O)[O-]</smiles>

Figure 8. Postulated transition states leading to the two enantiomers

The experimental and computed studies enable us to propose the following transition state in which the phosphonic acid activation leads to the formation of the opposite enantiomers compared to those obtained by Wennemers (Figure 8).

\section{Conclusions}

This study shows that peptide $\mathrm{H}-R$-Pro-S-Pro- $R$-pAla-OMe $\mathbf{I} \mathbf{a}_{11.5}$ is a catalyst with multifunctional activations. Covalent activation of carbonyl compounds through secondary amine and noncovalent activation by the phosphonic acid moiety allowed the 1,4-addition between aldehydes and nitroalkenes. NMR and modelisation studies confirmed the $(E)$-enamine formation while the presence of an acidic proton on the phosphonic acid seems to be crucial for the nitroalkene activation. The distance and the rigidity between both activating sites are also decisive for a good selectivity while replacing the central proline with a more flexible amino acid reduces the selectivities. Finally, the replacement of the carboxylic acid (a glutamic acid in Wennemer's catalyst) by a phosphonic acid has truly a striking impact in the reaction outcome, producing products with opposite configurations. These two groups have a strong influence in the transition state determining the final products, probably because the nitroalkene orientation is different in both catalytic pockets that differ in terms of nature and size.

\section{Experimental Section}

General Procedure for the Asymmetric Michael Reaction

$1 \mathrm{~mol} \%$ of catalyst was dissolved in methanol and chloroform $(1: 1)(20 \mathrm{~mL})$. trans- $\beta$-nitrostyrene $(1.0 \mathrm{mmol}, 1.0$ equiv. $)$ and propanal $(3.0 \mathrm{mmol}, 3.0$ equiv.) were added and the reaction mixture (homogeneous solution) was stirred at $0^{\circ} \mathrm{C}$. The reaction conversion was monitored by GC. After completion, the reaction mixture was concentrated under vacuum and the residue was 
dissolved in ethyl acetate. This solution was washed twice with water $(2 \times 20 \mathrm{~mL})$, dried over magnesium sulfate and concentrated to yield the desired product.

\section{Acknowledgements}

The Université Paris 13, Sorbonne Paris Cité, Centre National de la Recherche Scientifique (CNRS) and Ministère de l'Enseignement Supérieur et de la Recherche (MESR) are gratefully acknowlegded for financial support.

Keywords: organocatalysis • mechanism studies • Michael addition $\cdot$ DFT calculations $\cdot$ phosphonopeptide

[1] D. W. MacMillan, Nature. 2008, 455, 304-308.

[2] a) B. List, R. A. Lerner, C. F. Barbas, J. Am. Chem. Soc. 2000, 122 2395-2396; b) K. A. Ahrendt, C. J. Borths, D. W. C. MacMillan, J. Am Chem. Soc. 2000, 122, 4243-4244.

[3] For reviews of enamine and iminium catalysis, see: a) S. Mukherjee, J. W. Yang, S. Hoffmann, B. List, Chem. Rev. (Washington, DC, U. S.) 2007, 107, 5471-5569; b) A. Erkkilä, I. Majander, P. M. Pihko, Chem Rev. 2007, 107, 5416-5470.

[4] For selected general reviews for non-covalent activation mode, see: a) J.-F. Brière, S. Oudeyer, V. Dalla, V. Levacher, Chem. Soc. Rev. 2012 41, 1696; b) M. Mahlau, B. List. Angew. Chem. Int. Ed. 2013, 52, 518533; c) K. Brak, E. N. Jacobsen. Angew. Chem. Int. Ed. 2013, 52, 534561; d) A. Zamfir, S. Schenker, M. Freund, S. B. Tsogoeva, Org. Biomol. Chem 2010, 8, 5262-5276; for selected reviews on thiourea as H-bond donating catalyst, see: e) Z. Zhang, P. R. Schreiner, Chem Soc. Rev. 2009, 38, 1187-1198; f) M. S. Taylor, E. N. Jacobsen, Angew. Chem. Int. Ed. 2006, 45, 1520-1543; g) M. Terada, Bull. Chem. Soc. Jpn. 2010, 83, 101-119; h) S. Schenker, A. Zamfir, M. Freund S.B. Tsogoeva, Eur. J. Org. Chem. 2011, 2209-2222; i) D. Parmar, E. Sugiono, S. Raja, M. Rueping, Chem. Rev. 2014, 114, 9047-9153.

[5] a) B. List, J. Am. Chem. Soc. 2000, 122, 9336-9337; b) A. Ting, S. E. Schaus, Eur. J. Org. Chem. 2007, 5797-5815; c) J. M. Verkade, L. J. van Hemert, P. J. Quaedflieg, F. P. Rutjes, Chem. Soc. Rev. 2008, 37, 29-41; d) M. Wiesner, J. D. Revell, H. Wennemers, Angew. Chem., Int. Ed. 2008, 47, 1871-1874; e) N. Halland, A. Braunton, S. Bachmann, M. Marigo, K. A. Jørgensen, J. Am. Chem. Soc. 2004, 126, 4790-4791; f) X. Fan, S. Sayalero, M. A. Pericàs, Adv. Synth. Catal. 2012, 354, 29712976; g) Y. Hayashi, S. Aratake, Y. Imai, K. Hibino, Q. Y. Chen, J. Yamaguchi, T. Uchimaru, Chem. Asian J. 2008, 3, 225-232; h) D. Enders, C. Grondal, M. R. Huttl, Angew. Chem., Int. Ed. 2007, 46, 1570-1581; i) R. Marcia de Figueiredo, M. Christmann, Eur. J. Org Chem. 2007, 2575-2600; j) K. S. Halskov, B. S. Donslund, S. Barfusser, K. A. Jorgensen, Angew. Chem., Int. Ed. 2014, 53, 4137-4141; k) J. McNulty, C. Zepeda-Velazquez, Angew. Chem., Int. Ed. 2014, 53, 8450-8454; I) T. Akiyama, J. Itoh, K. Yokota, K. Fuchibe, Angew. Chem., Int. Ed. 2004, 43, 1566-1568; m) D. Uraguchi, M. Terada, J. Am. Chem. Soc. 2004, 126, 5356-5357.

[6] For selected examples of bioactive molecule syntheses by organocatalysis, see: a) R. Marcia de Figueiredo, M. Christmann, Eur. J. Org. Chem. 2007, 2575-2600; b) E. Marqués-López, R. P. Herrera, M. Christmann, Nat. Prod. Rep. 2010, 27, 1138-1167; c) S. B. Jones, B. Simmons, A. Mastracchio, D. W. C. MacMillan, Nature 2011, 475, 183188; d) Z. Zhang, J. C. Antilla, Angew. Chem. Int. Ed. 2012, 51, 1177811782; e) M. E. Abbasov, D. Romo, Nat. Prod. Rep. 2014, 31, 13181327. f) Y. Hayashi, S.Ogasawara, Org. Lett. 2016, 18, 3426-3429. g) M. Candy, T. Durand, J.-M. Galano, C. Oger, Eur. J. Org. Chem. 2016, 5813-5816.
[7] a) S. Inoue, Adv. Polym. Sci. 1976, 21, 78-106; b) J.-I. Oku, N. Ito, S Inoue, Makromol. Chem. 1979, 180, 1089-1091; c) M. S. Iyer, K. M. Gigstad, N. D. Namdev, M. Lipton, J. Am. Chem. Soc. 1996, 118, 49104911; d) T. E. Horstmann, D. J. Guerin, S. J. Miller, Angew. Chem., Int. Ed. 2000, 39, 3635-3638; e) E. R. Jarvo, S. J. Miller, Tetrahedron Lett. 2002, 58, 2481-2495; f) A. Berkessel, Curr. Opin. Chem. Biol. 2003, 7, 409-419; g) S. J. Miller, Acc. Chem. Res. 2004, 37, 601-610; h) S. M. Mennen, J. T. Blank, M. B. Tran-Dube, J. E. Imbriglio, S. J. Miller, Chem. Comm. 2005, 195-197. i) Y. Arakawa, M. Wiesner, H. Wennemers, Adv. Synth. Catal. 2011, 353, 1201-1206; j) J. Li, G. Yang, Y. Qin, X. Yang, Y. Cui, Tetrahedron: Asymmetry 2011, 22, 613-618; k) X. Fan, S. Sayalero, M. A. Pericàs, Adv. Synth. Catal. 2012, 354, 29712976; I) L. Tuchman-Shukron, S. J. Miller, M. Portnoy, Chem. Eur. J. 2012, 18, 2290-2296; m) K. S. Feu, A. F. de la Torre, S. Silva, M. A. F. de Moraes Junior, A. G. Corrêa, M. W. Paixão, Green Chem. 2014, 16, 3169-3174; n) C. Rodríguez-Escrich, M. A. Pericàs, Eur. J. Org. Chem. 2015, 1173-1188.

[8] a) E.R. Jarvo, S. J. Miller. Tetrahedron 2002, 58, 2481-2495; b) S. J. Miller. Chem. Res. 2004, 37, 601-610; c) E. A. Colby Davie, S. M. Mennen, Y. Xu, S. J. Miller. Chem. Rev. 2007, 107, 5759-5812.

[9] a) P. Krattiger, R. Kovasy, J. D. Revell, S. Ivan, H. Wennemers, Org. Lett. 2005, 7, 1101-1103 b) M. Wiesner, H. Wennemers, Synthesis, 2010, 9, 1568-1571.

[10] a) M. Wiesner, M. Neuburger, H. Wennemers, Chem. Eur. J. 2009, 15, 10103-10109; b) F. Bächle, J. Duschmalé, C. Ebner, A. Pfaltz, H. Wennemers, Angew. Chem. Int. Ed. 2013, 52, 12619-12623.

[11] We define our modified amino-acid $\mathrm{H}-\mathrm{AA}_{1}-\mathrm{OMe}$ bearing a phosphonic acid as pAla for phosphonoalanine.

[12] M. Cortes-Clerget, O. Gager, M. Monteil, J.-L. Pirat, E. Migianu-Griffoni, J. Deschamp, M. Lecouvey, Adv. Synth. Catal. 2016, 358, 34-40.

[13] $11.5,6.6$ and 2.3 refer to the $\mathrm{pH}$ adjustment during the catalyst preparation. 11.5 refer to the all-deprotonated catalyst while 6.6 refer to the $\mathrm{NH}_{2}^{+} / \mathrm{OH} / \mathrm{O}^{-}$form and 2.3 refer to the all-protonated catalyst.

[14] The syntheses of la-d and lla-d were reported in our previous work (ref 12). IIla-d and IVa-d were synthesized starting from aspartic acid and glutamic acid respectively according to the same strategy than la-d and lla-d. For more details, see supporting information.

[15] M. Wiesner, G. Upert, G. Angelici, H. Wennemers. J. Am. Chem. Soc 2010, 132, 6-7.

[16] Initial rates were determined by GC. For more details, see Supporting information.

[17] C. Puchot, O. Samuel, E. Duiiach, S. Zhao, C. Agami, H. B. Kagan, J. Am. Chem. Soc. 1986, 108, 2353-2357.

[18] L. Hoang, S. Bahmanyar, K. N. Houk, B. List, J. Am. Chem. Soc. 2003, 125, 16-17.

[19] Blackmond interpreted the first results by a solubility issues for more details see a) M. Klussmann, H. Iwamura, S. P. Mathew, D. H. Wells, Jr., U. Pandya, A. Armstrong, D. G. Blackmond, Nature 2006, 441, 621623 b) M. Klussmann, S. P. Mathew, H. Iwamura, D. H. Wells, Jr., A Armstrong, D. G. Blackmond, Angew. Chem. Int. Ed. 2006, 45, 79897992.

[20] For more details, see Supporting Information

[21] Preliminary Mass experiment were performed and the two new species were detected at $\mathrm{m} / \mathrm{z}=416$ (enamine) and $\mathrm{m} / \mathrm{z}=417$ (iminium intermediate). For more details see Supporting Information.

[22] J. E. Hein, J. Bures, Y. H. Lam, M. Hughes, K. N. Houk, A. Armstrong, D. G. Blackmond, Org. Lett. 2011, 13, 5644-5647.

[23] M. Hatano, K. Moriyama, T. Maki, K. Ishihara, Angew. Chem. Int. Ed. 2010, 49, 3823-3826. 
Entry for the Table of Contents (Please choose one layout)

Layout 1:

\section{FULL PAPER}

One catalyst to rule them all. Mechanistic investigations were performed in order to unveil the activation modes and catalytic efficiency of the H-Pro-Pro-pAla-OMe catalyst in the 1,4-addition between aldehydes and nitroalkenes. This tripeptide behaves as a multiactivating catalyst, able to interact through a secondary amine group and noncovalent activations. A restrained tripeptide is optimal for better selectivities and fast reaction.

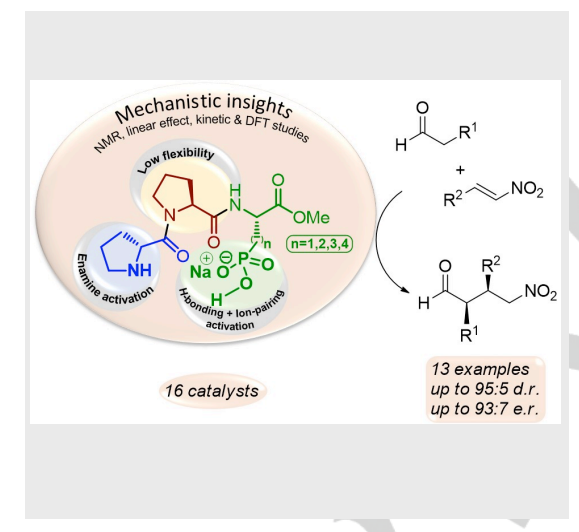

Margery Cortes-Clerget, Jesús Jover, Jade Dussart, Emilie Kolodziej, Maelle Monteil, Evelyne Migianu-Griffoni, Olivier Gager, Julia Deschamp, * Marc Lecouvey*

Page No. - Page No.

Bifunctional Tripeptide Including a Phosphonic Acid as Brønsted Acid for Michael Addition: Mechanistic Insights 